\title{
Treatment of Human Immunodeficiency Virus-1: Current Challenges and Future Perspectives
}

Inês Pereira, Cristina Luxo and Ana Miguel Matos*

Laboratory of Virology, Faculty of Pharmacy, University of Coimbra, Portugal

\begin{abstract}
Today, HIV-1 infection maintains its status as a grave public health problem. The development of effective antiretroviral drugs has greatly increased life expectancy of infected patients by arresting disease progression, but treatment is not able to eliminate the virus from the body, requiring lifelong adherence to prevent viral rebound and its consequent damage on the host immune system. The ability of the virus to remain latent in cellular reservoirs is a major obstacle in the development of curative therapies, in part because there is still a considerable lack of understanding of viral latency processes. A lot of research has been conducted to study and clarify such mechanisms and propose strategies to eradicate the virus (sterilising cure) or at least permanently control it (functional cure) in order to bypass the need for chronic antiretroviral therapy.

The purpose of this literature review is to discuss the state of the art in HIV-1 research, with a particular focus on the mechanisms by which HIV-1 eradication has thus far remained an unattainable goal. Future perspectives for the development of a functional or sterilising cure to HIV-1 infection will also be explored.
\end{abstract}

Keywords: HIV-1; Latency; Reservoirs; Functional cure; Sterilising cure; Eradication strategies

\section{Introduction}

Despite the incredible advances in therapeutic regimens since treatment first became available, HIV/AIDS is still a global health threat, maintaining its status as one of the deadliest viral diseases in human history. The WHO points to a total number of 1.1 million deaths by AIDS and close to 37 million people living with HIV worldwide in 2015. Sub-Saharan Africa accounts for a great majority of these 37 million and represents nearly $70 \%$ of the global total number of new infections [1]. Repeated failures in developing prophylactic vaccines have also been disappointing, as a definite means of prevention was initially expected to be discovered within a couple of decades.

Benjamin Franklin, American author, scientist and inventor said, "An ounce of prevention is worth a pound of cure", and in the case of HIV-1, this statement certainly holds true. This virus is transmitted through direct contact of certain bodily fluids such as blood, semen, vaginal secretions and breast milk. Risk behaviours like unprotected sex and the sharing of needles and syringes between drug users are the two top causes of HIV-1 infection in developed countries, where education on how to prevent contamination can contribute to reduce the spread of the disease. Such measures may, however, not yield such positive results in the underdeveloped world, where the lack of safe sex practices and access to healthcare represents an enormous obstacle to the fight against HIV-1 transmission. While mother to child transmission is uncommon in the developed world, where proper treatment and care of both mother and baby can prevent contamination of the latter, it is still one of the main causes of infection in developing countries.

According to UNAIDS [1], more than half of the total number of people infected with HIV worldwide are still not receiving treatment, and many of them do not know they're infected. To reduce the impact of the disease on a global scale, treatment must be made available worldwide, since it is responsible for massive improvements in quality of life as well as longevity, while simultaneously contributing to reduce the risk of transmission and thereby minimizing the spread of the disease.
Prior to the introduction of combination antiretroviral therapy (cART), also known as highly active antiretroviral therapy (HAART), HIV-1 was responsible for the death of the vast majority of the infected individuals. There was no effective way to control infection and patients would soon reach the immunodeficiency stage and develop deadly opportunistic infections [2]. Due to the development of effective drugs that stunt viral replication, carriers of the virus who are subjected to cART and comply with treatment can now have an expectancy of life similar to that of healthy individuals [3], meaning that HIV/AIDS can now be classified as a chronic illness rather than a lethal one.

Despite the positive clinical outcomes observed in patients with HIV-1 under CART, they do not represent a cure. Currently available antiretroviral drugs do not fully eliminate the virus from the body, so in the event that treatment is interrupted, patients quickly experience resurgence of viraemia [4]. HIV-1 has the ability to remain inactive in a latent form in certain cells, escaping detection by both cART and the host immune system. Under specific conditions, viral transcription is activated and new virions, capable of propagating infection, are released into the bloodstream. Therefore, lifelong treatment must be enforced to avoid uncontrolled viral replication and de novo infection of susceptible cells.

Many advances have been made in this area, but many questions surrounding HIV-1 remain unanswered today. Full eradication of the virus, the holy grail of HIV-1 research, appears to be mechanistically implausible. Because there is a lack of clear understanding of the

*Corresponding author: Ana Miguel Matos , Laboratory of Virology, Faculty of Pharmacy, Pólo das Ciências da Saúde, Azinhaga de Santa Comba, University of Coimbra , 300-548 Coimbra, Portugal, Tel: +351 239488446; +351 963038110; E-mail: anamatos@ci.uc.pt

Received July 06, 2016; Accepted July 15, 2016; Published July 22, 2016

Citation: Pereira I, Luxo C, Matos AM (2016) Treatment of Human Immunodeficiency Virus-1: Current Challenges and Future Perspectives. J AIDS Clin Res 7: 603. doi:10.4172/2155-6113.1000603

Copyright: ( 2016 Pereira l, et al. This is an open-access article distributed under the terms of the Creative Commons Attribution License, which permits unrestricted use, distribution, and reproduction in any medium, provided the original author and source are credited. 
processes behind latency and viral infection, research must continue in order to clarify whether or not the complete elimination of the virus from infected patients is an achievable goal. Alternative strategies to at least provide better control of infection should also be investigated.

This literature review aims to discuss current trends in HIV-1 cure research, focusing on the implications of complex mechanisms of viral latency on the development of effective eradication strategies. Recent advances in this area will be explored as well as future directions of HIV-1 investigation towards HIV-1 cure or remission.

\section{Basics of HIV-1 Infection}

\section{Morphology}

HIV- 1 consists in two positive sense single-stranded RNA molecules, packaged in a truncated conical capsid and surrounded by an outer lipid envelope punctuated by glycoproteic spikes. The glycoproteins that make up the envelope consist, in turn, of two subunits: gp120, the surface domain and gp41, the transmembrane domain [5].

\section{Replication cycle}

HIV-1 enters permissive cells through specific binding of its surface and transmembrane proteins to their receptor and co-receptor. The viral receptor of HIV-1 is CD4, which is present on the surface of T-helper cells, B-lymphocytes, macrophages, dendritic cells and brain cells. To enter the cell and cause infection, the virus must also bind to a co-receptor, normally chemokine receptors such as CXCR4 or CCR5. T-cell tropic viruses use CXCR4 (T-tropic) while macrophagetropic (M-tropic) viruses primarily use CCR5, although several other HIV-1 co-receptors have been identified. While gp120 binds to CD4 and the co-receptor on the host cell's surface, gp41 mediates membrane fusion and allows the viral particle to enter the cytoplasm of the cell [5]. HIV-1 has the ability to integrate its genome into the host cell genome, originating a provirus. After entering the cytoplasm of the cell and undergoing uncoating, the viral enzyme reverse transcriptase (RT) works to produce a complementary DNA strand from the original viral positive RNA strand, resulting in a hybrid RNA-DNA molecule. The enzyme then degrades the RNA strand, due to its ribonuclease activity, and finally generates the complementary DNA strand using the previously created DNA strand as template, acting as DNA-dependent DNA polymerase. This double-stranded DNA (dsDNA) intermediate is transported to the nucleus, where it can integrate the cell genome with the help of integrase, another viral enzyme [6]. Consequently, whenever the cellular mechanisms of transcription and translation are activated, not only are host cell proteins produced, but also viral ones. The transcribed viral mRNA, identical to the original positive single-stranded RNA, is translated into viral proteins. A complex process of assembly occurs, involving viral and cellular components alike, culminating in the release of new virions, which become infectious after a maturation stage.

\section{Stages of infection}

Infection with HIV-1 can be divided into three stages: primary infection, clinical latency and AIDS. During primary infection, there is an initial high level of viral RNA in plasma, as the virus replicates freely after infecting its target $\mathrm{CD} 4^{+} \mathrm{T}$ cells. Consequently, T-helper cell count begins by declining, but increases after resolution of the primary infection by virtue of the mechanisms involved in suppressing viral replication once the patient's immune system recognises and attempts to fight the virus [5]. Clinically, acute retroviral syndrome can occur. This syndrome is characterised by influenza-like symptoms such as fever, headache, myalgia and fatigue. It sets in about two to four weeks, but only in about $50 \%$ of the patients.

During the clinical latency phase, there are no symptoms associated with viral replication. The patient's immune response brings viraemia to a stable level, called the viral set point [6]. However, because replication is ongoing in the absence of treatment, the viral load rises with an associated decrease in $\mathrm{CD}^{+}{ }^{+} \mathrm{T}$ lymphocyte count. As the host immune system gradually becomes more impaired by cell destruction, the risk of developing opportunistic infections continues to increase, and eventually a third stage of infection is established: Acquired immunodeficiency syndrome, or AIDS. This stage is reached when $\mathrm{CD} 4^{+} \mathrm{T}$ cell count is as low as 200 per cubic millimetre or represents under $14 \%$ of the total number of lymphocytes, or, alternatively, an AIDS-defining illness is present. The latter is defined as an opportunistic infection so rare that only causes disease in severely immunocompromised patients.

If a person who is infected with the virus does not receive treatment, viraemia remains uncontrolled. Their immune system is not strong enough to stave off infection, and as an increasing number of $\mathrm{CD} 4^{+} \mathrm{T}$ cells are destroyed [7], the patient becomes progressively more susceptible to the development of lethal opportunistic infections.

\section{Current Challenges}

\section{Available therapies and their disadvantages}

The different classes of drugs used in combination therapy of HIV-1 all block a specific process in viral replication: fusion inhibitors and CCR5 co-receptor antagonists inhibit viral entry, nucleoside reverse transcriptase inhibitors (NRTIs) and non-nucleoside reverse transcriptase inhibitors (NNRTIs) prevent reverse transcription of the genome, integrase inhibitors block viral integration and protease inhibitors (PIs) hinder viral maturation [4]. The combination of drugs from different classes effectively blocks replication and therefore lowers viraemia. This allows patients to live relatively normal lives while contributing to minimise the probability of HIV-1 transmission. The development of technological means to combine several drugs in the same formulation was also advantageous, as it resulted in enhanced treatment adherence, which greatly benefits public health in addition to the improvement in the individual's own quality of life.

The inhibition of replication by cART can lower the patients' viraemia so effectively that it becomes undetectable using conventional methods $(<50$ copies $/ \mathrm{mL})$, but in the event that treatment is interrupted, it quickly becomes measurable again, which indicates that current therapy is not able to completely eliminate HIV-1. It has been shown that a residual viraemia can be detected using ultrasensitive assays in patients undergoing cART [2]. The reason for this behaviour is still object of debate. Lymphoid tissue, where drug penetration is less effective, may represent a sanctuary for HIV-1 replication and could be responsible for the maintenance of low-level viraemia [8]. Another important factor in the reactivation of HIV-1 infection is that while these drugs are able to stunt viral replication, they cannot eliminate transcriptionally silent virus integrated in resting $\mathrm{CD} 4^{+} \mathrm{T}$ cells and perhaps even other unidentified cells [3]. If the host cell is activated, viral replication is bound to restart, hence the rise in viraemia in the absence of treatment.

These are not the only negative aspects of cART. Chronic, lifelong therapy is expensive, making it difficult to set up in underdeveloped countries. Although cART can greatly reduce the chances of in utero and breast milk transmission, there are many obstacles to its implementation in resource-limited countries, making vertical 
transmission the top cause of infection in such regions. Moreover, there are questions of whether or not cART may be responsible for increased susceptibility to coronary disease, cancer, liver and kidney dysfunction and neurologic impairment [9] in the end. Persistent inflammation even in the presence of cART is likely to contribute to this phenomenon [8]. Finally, several very resistant strains of HIV-1 have been isolated from patients undergoing treatment [6].

\section{The role of HIV-1 latency in treatment limitations}

It was initially thought that cART would eventually lead to full eradication of the virus. Because infected cells have a short half-life due to viral cytopathic effects and clearance by the immune system, simply administering cART should mean that there would come a time the virus would completely disappear from the body, since previously infected cells would ultimately degenerate and die. However, this theory quickly became obsolete as a new population of $\mathrm{T}$ cells that is characterised by a very long half-life was found to allow for viral persistence [10]. These cells, called memory $\mathrm{T}$ cells, are at least partly responsible for the rebound in viraemia that is observed in seropositive patients when cART is interrupted.

HIV-1 replication depends on the activation state of the infected cell, mainly because it requires transcription factors, such as NF- $\kappa B$ and NFAT, which are absent from the nucleus in resting cells [10], rendering them non-permissive for viral gene expression. Thus, latency is established when, after an initial activation of the $\mathrm{T}$ cell by its specific antigen, it transitions back to a resting state. HIV-1, having integrated into the host genome, becomes transcriptionally silent in its reservoir, and remains in a quiescent state until the cell is reactivated [4]. Therefore, viral latency can be seen as an accidental consequence of HIV-1 tropism for activated $\mathrm{CD} 4^{+} \mathrm{T}$ cells [7], which can become infected as they revert to a resting state, leaving the virus unable to replicate.

Resting cells are, by definition, transcriptionally inactive. HIV-1 is not translated into viral proteins, and so it does not leave a mark on the cell to help the host immune system detect that the cell is infected. This means that not only are infected cells able to escape the influence of cART, as previously stated, but also of the host immune system. When stimulated, they are reactivated. Viral production is resumed, which, in the absence of treatment, results in a rise in viraemia and de novo infection of susceptible cells [10].

While resting memory $\mathrm{CD} 4^{+} \mathrm{T}$ cells are the only well-characterised source of residual viraemia responsible for viral rebound, they are unlikely to be the only cell type capable of contributing to the quiescent reservoir. Other cell populations permissive for HIV-1 entry, such as dendritic cells, monocytes/macrophages and haematopoietic progenitor cells, among others, have been proposed as sources of viral production [4], although no indisputable evidence has been found of their implication in viral latency so far.

A possible source of residual viraemia is ongoing low-level viral replication in $\mathrm{CD} 4^{+} \mathrm{T}$ cells in the presence of treatment. Several studies have argued against this theory. Intensification of cART does not seem to lower viraemia any further below its vestigial level and there are no genetic differences in the residual viral population to suggest that replication continues to occur during treatment [11]. Instead, the homogeneity of these clones seems to indicate that viraemia persists due to the reactivation of provirus-containing resting cells, and that cART does effectively block replication [4].

However, as previously mentioned, certain anatomical sites, such as the central nervous system, gut-associated lymphoid tissue, brain and genital tract, can act as viral sanctuaries [9]. Tissues where drug penetration is inadequate constitute a barrier to complete HIV-1 elimination, as viral replication is not fully inhibited due to suboptimal antiretroviral drug concentrations [12]. A recent study conducted by Lorenzo-Redondo et al. [13] suggests that low-level ongoing replication in lymphoid tissue may be responsible for the replenishment of viral reservoirs and that drug resistance does not develop because antiretroviral drug concentration in those tissues is not high enough to confer competitive advantage to resistant strains. This study makes a compelling argument in favour of this controversial hypothesis. It is unknown whether the reactivation of resting cells happens often enough to maintain low-level viraemia, and replication in compartments other than the blood may explain this phenomenon [9].

If cell types other than $\mathrm{CD} 4^{+} \mathrm{T}$ cells can indeed become chronically infected, they are likely to play a critical role in the maintenance of residual viraemia through ongoing low-level replication. It has been shown that antiretroviral drugs, namely NRTIs and PIs, are less effective in monocytes and macrophages than in lymphocytes [2]. Inhibition of viral replication occurring in these cells as well as in other cell types may be incomplete, and so they may constitute sources of residual viraemia. Identification of such cells is therefore an important step towards the development of eradication strategies. Studies suggest that monocytes, macrophages and dendritic cells can harbour proviruses and may have an important role in viral persistence, particularly in sanctuary sites where low-level infection is possible [14]. Whether or not these cell types allow for true viral latency is, as previously mentioned, unknown.

Regardless of the origin of this residual viraemia, future attempts to develop effective treatment for HIV-1 must take into consideration that it will only be possible to eliminate the virus from all of its reservoirs if latency is aborted or, alternatively, the provirus is removed from existing memory cells. Further improvements in cART such as penetration enhancement or treatment intensification might help lessen the problem in sanctuary sites, but they will have no effect on eliminating latent virus from its cellular reservoirs [9], which means that entirely new strategies must be designed for complete viral eradication to be achieved.

\section{Towards a Cure: Full Eradication vs. Viral Remission}

When discussing the possibility of curing HIV-1, it is important to define what exactly is meant by "cure". A sterilising cure, as the name itself indicates, is one that manages to deprive the body entirely of the virus; that is, one that is able to completely eradicate all HIV-1-infected cells, including the viral latent reservoirs. It thus becomes clear that it is crucial to fully understand the mechanisms behind HIV-1 latency before attempting to identify and develop therapeutics that can provide a sterilising cure [12]. The only instance where a patient has been healed of HIV-1 via a sterilising cure (the Berlin patient) will be discussed further below.

A functional cure, on the other hand, is one in which viral replication is controlled by the host immune system, preventing disease progression and transmission in the absence of cART [10]. This situation actually occurs naturally in a small percentage of patients with HIV-1. These patients, designated elite controllers (ECs), are HIV-1positive individuals who are able to maintain viraemia below detection limits without the use of antiretroviral therapy [2], after experiencing low peak viral loads during primary infection [15]. Research has shown that many ECs are infected with replication-competent viruses, capable of causing immunodeficiency in other hosts [10]. This seems 
to suggest that, in most cases, ECs owe this phenomenon to particular host characteristics rather than to faults in HIV-1 replication, which would make it less virulent and therefore limit its ability to destroy the host immune system.

Because of this extraordinary behaviour, these patients can be considered models of functional cure. The fact that infection is greatly contained by their immune system alone, in the absence of cART, enables all undesirable effects of current therapies to be bypassed, as well as other negative aspects such as the need for chronic medication, which is a great advantage for the patient. Learning the mechanisms behind the uniquely effective performance of the immune system of ECs is likely to provide essential tools for the development of a functional cure.

Quite a few theories have been proposed to explain this behaviour, but one is particularly interesting. Studies conducted in ECs have shown that many of these patients have protective HLA class I alleles, namely $\mathrm{B}^{\star} 27$ and $\mathrm{B}^{\star} 57$, which present critical conserved HIV-1-specific epitopes to $\mathrm{CD}^{+} \mathrm{T}$ cells, leading to effective cytotoxic $\mathrm{T}$ lymphocyte (CTL) responses that result in the death of infected cells [15]. However, not all ECs have these alleles or this sort of strong response, not everyone with these alleles becomes an EC [10], and so some aspects of elite control remain unexplained.

Administration of cART in the early stages of HIV-1 infection seems to sometimes lead to a sort of "temporary" functional cure, in which a patient who undergoes aggressive therapy during primary infection can interrupt cART and not suffer rebound viraemia for a certain period. Similarly, to what happens in ECs, viral replication is controlled to the point where HIV-1 RNA levels are undetectable in plasma, and so these patients are called post-treatment controllers [15]. However, this effect is transitory.

Because ECs have low viraemia levels in primary infection and these post-treatment controllers often presented with high viral loads as well as acute retroviral syndrome before starting cART, it does not seem likely that they would become ECs. The mechanisms behind posttreatment control are still unknown, as none of the theories proposed to explain this phenomenon have been convincing; the alleles involved in elite control also do not play a role in this case [10]. Discovering how both of these situations occur would be half the battle to learn how to recreate them and develop a functional cures under the shape of a therapeutic vaccine, for example.

In 2010, an infant born to an HIV-1 positive mother who did not undergo treatment during pregnancy began aggressive cART 30 hours after birth. Known as the Mississippi baby, the newborn was presumed to have been infected with HIV-1 as the result of in utero transmission. A diagnosis was confirmed after repeat testing, as the baby presented with high levels of HIV-1 RNA [16]. Therapy was discontinued at 18 months of age, but no rebound was observed until more than two years afterwards. Although the child was not cured, the long period during which HIV-1 levels remained undetectable after interrupting cART indicates that early therapy may have contributed to limit the size of the reservoir. By blocking viral replication soon after transmission, fewer cells become infected and, consequently, productive infection originating from viral reservoirs occurs at a slower rate when treatment is stopped [17]. This is consistent with the behaviour observed in posttreatment controllers.

In 2015, another case of remission was reported in an 18-year old French girl who started treatment at birth but discontinued it at 6 years of age. After twelve years without cART, she remains aviraemic. This is the longest case of remission ever reported, which brings renewed optimism to HIV-1 cure research [18].

All of these observations (Table 1) suggest that viral reservoirs are smaller in patients who begin cART during primary infection than in those who only start therapy in the chronic phase of infection. As summarised by Passaes and Sáez-Cirión [8], timing and duration of treatment seem to be important factors in the establishment of longlasting HIV-1 control, and success may be dependent on baseline viral load as well as immune activation.

Although a functional cure seems more realistic to achieve, it cannot be considered ideal. The drawback of having an overactive immune system is that ECs also develop chronic inflammation and are therefore much more likely to suffer from non-AIDS events than healthy individuals [2]. Such events include cardiovascular disease, neuropsychiatric disorders and bone-related illnesses [11], as well as renal and liver disease and non-AIDS malignancies [9]. Thus, research should continue attempting to devise strategies to eradicate the virus from the body, in order to obtain a sterilising cure. This will only be possible once the mechanisms behind HIV-1 latency are fully understood. Some strategies have so far been proposed, but only once has a sterilising cure been observed, and it is likely to remain a single isolated case.

\section{The Berlin patient - Proof of concept?}

When, in 2007, Timothy Brown received a hematopoietic stem cell (HSC) transplant for the treatment of cancer, he did not expect to be the first person to ever be cured of HIV. The HIV-1-positive patient, diagnosed with acute myeloid leukaemia, received a double allogeneic HSC transplant from a donor screened for homozygosity for the CCR5 $\triangle 32$ allele. This mutation, responsible for a $32 \mathrm{bp}$ deletion in the CCR5 gene, was expected to have some sort of effect in the infection, as CCR5 is one of the main co-receptors used by HIV-1 to enter susceptible cells. The aforementioned mutation would prevent viral entry into cells by M-tropic viruses, as they would not express CCR5 [15]. Indeed, the Berlin patient, as he is known, has now gone nine years without detectable levels of HIV-1 in blood and tissue samples since he first discontinued cART in preparation for the transplant [8].

The use of HSC transplantation to cure HIV-1 cannot be perceived as a feasible, commonplace strategy due to its innumerable risks and costs, as well as the difficulty in finding compatible donors. This approach has only been object of study in seropositive patients with concurrent leukaemia and/or lymphoma. Despite the promising results obtained with the Berlin patient, this has so far been the only instance in which HSC transplantation resulted in complete viral eradication. Two more patients who received HSC transplants from wild-type CCR5 donors, known as the Boston patients, continued on cART and managed to

\begin{tabular}{|c|c|c|c|c|}
\hline & Time of cART initiation & Duration of cART & Duration of virologic control after cART interruption & References \\
\hline Mississippi baby & 30 hours after birth & 18 months & 27 months & {$[16,17]$} \\
\hline Post-treatment controllers & During primary infection & Median of 3 years & Median of 7 years & {$[8]$} \\
\hline French teenager & At 3 months of age (after a 6 week course of zidovudine) & 6 years & No rebound observed after 12 years & [18] \\
\hline
\end{tabular}

Table 1: Significant cases of sustained virologic control after treatment interruption. 
Citation: Pereira I, Luxo C, Matos AM (2016) Treatment of Human Immunodeficiency Virus-1: Current Challenges and Future Perspectives. J AIDS Clin Res 7: 603. doi:10.4172/2155-6113.1000603

Page 5 of 9

obtain undetectable levels of HIV-1, but suffered a strong rebound in viraemia within a year of interrupting cART. It is worth noting that, like the Berlin patient, these two patients were heterozygous for the CCR5 $\Delta 32$ mutation, which does not confer resistance to infection as homozygosity does but may delay disease progression, which may have contributed to stall the inevitable rebound [15]. A few other patients died from treatment complications, including graft-versus-host disease (GVHD), while others simply never reached the same undetectable levels of HIV-1 in blood as the Berlin and the Boston patients did, which means the viral reservoirs remained unaffected following transplantation [8].

It is thought that radiation therapy, which initially decimated Brown's immune system and GVHD, which he contracted after transplantation, both had a big role in clearing out the viral reservoirs from his body, and may have greatly contributed to his extraordinary recovery (Figure 1) [19]. Whether or not viral persistence remains in this patient is unknown. Even if the latent reservoirs were not completely eliminated in the process, the resistant donor-derived cells lacking CCR5 would block de novo infection of susceptible cells [10], although a change in viral tropism could lead to future resurgence of viraemia [15]. Any occasional reactivation of infected resting $\mathrm{T}$ cells would activate viral replication, originating changes in the viral genetic information that could eventually render the virus able to infect other types of cells through different co-receptors. Fortunately, for the patient, there is currently no indication of this situation having occurred.

Most of the factors involved in this case, if not all of them, are impossible to replicate, and so a cure remains elusive. Nevertheless, it provides proof of concept that it may be possible to eradicate HIV-1, and represents a kindle of hope that one day a sterilising cure will be available.

\section{Future Strategies}

The experience of the past thirty years has allowed the scientific and medical community to devise innovative strategies (Table 2) to eliminate the virus from the body of infected individuals to effectively cure them from HIV-1, although they have met with little success so far.

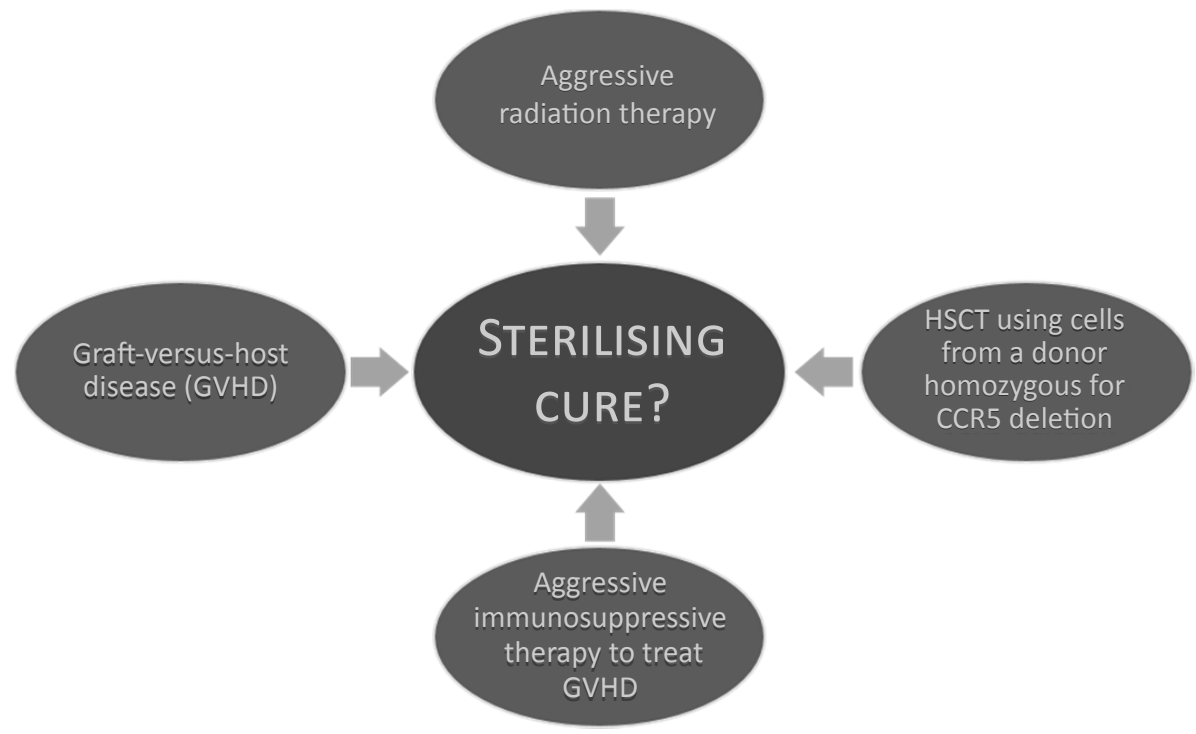

Figure 1: Factors that may have contributed to full eradication in the Berlin patient case.

\begin{tabular}{|c|c|c|}
\hline \multicolumn{2}{|r|}{ Strategies } & \multirow{2}{*}{$\begin{array}{c}\text { References } \\
{[8,14]}\end{array}$} \\
\hline "Shock-and-kill” & Histone deacetylases (HDAC) inhibitors (vorinostat, panobinostat) & \\
\hline & Protein kinase C (PKC) agonists (prostratin, bryostatin-1) & [19] \\
\hline & Disulfiram & [14] \\
\hline & BRD4 inhibitors (JQ1) & {$[8]$} \\
\hline & Hexamethylene bisacetamide (HMBA) & [11] \\
\hline & Host transcription repressor inhibitors & [11] \\
\hline \multirow[t]{5}{*}{ Gene therapy } & Zinc-finger nucleases & {$[3,9,25]$} \\
\hline & CRISPR/Cas9 & [26] \\
\hline & Ribozymes & {$[3,11,15]$} \\
\hline & SiRNA & {$[3,11,15]$} \\
\hline & Antisense anti-miRNA & {$[2,11]$} \\
\hline Permanent suppression of viral transcription & Transcription inhibitors & [22] \\
\hline \multirow[t]{4}{*}{ Immunotherapy } & Therapeutic vaccines & {$[4,19]$} \\
\hline & Downregulation of immunoregulatory molecules (PD-1) & {$[3,27]$} \\
\hline & Broadly neutralizing antibodies (bNAbs) & [27] \\
\hline & HIV-1 targeted immunotoxins & [4] \\
\hline
\end{tabular}

Table 2: Possible approaches to improve HIV-1 treatment. 
Viral latency is maintained by a series of complex mechanisms, including modification of the chromatin environment, downregulation of transcription factors and suppression of viral mRNA [15], among others. HIV-1 cure research has focused on enlightening the processes behind such mechanisms and identifying potential therapeutic targets for the future development of therapies that may hopefully result in either a functional or a sterilising cure.

\section{"Shock-and-Kill" strategies using latency-reversing agents}

One of the most widely discussed strategies to eradicate HIV-1 involves purging the latent reservoir. This approach consists in using small molecules that activate HIV-1 transcription to reactivate viral expression in resting cells (the "shock"). Active viral replication leads to cell death, either by viral cytopathic effects or by immune clearance due to the incorporation of viral proteins on the cell membrane, which means that latent cells should be eliminated once viral replication is initiated, resulting in a reduction in the size of the reservoir. However, because these processes are not sufficient to completely remove these cells, immune strategies that stimulate cytotoxic T lymphocyte (CTL) responses such as therapeutic vaccines or immunotoxins are likely to be necessary to aid in the clearance (the "kill") [20]. Antiretroviral therapy would be ongoing during this entire process, preventing susceptible cells from becoming infected by virus released from reactivated cells.

An initial approach to latency reversal consisted in inducing global $\mathrm{T}$-cell activation in order to reactivate viral production. However, this resulted in toxic levels of immune activation due to the release of large amounts of pro-inflammatory cytokines, so recent attempts to purge the reservoir have focused on molecules that can activate viral transcription without activating all resting cells [4]. These latencyreversing agents (LRAs) include several groups of compounds such as histone deacetylase (HDAC) inhibitors, protein kinase C (PKC) agonists and bromodomain inhibitors.

At the transcriptional level, chromatin organisation and epigenetic modifications as well as sequestration of host transcription factors are a few of the strategies the virus takes advantage of to become stably integrated in a state of latency.

Modification of the chromatin environment: Histones, which form a complex with DNA called chromatin, have a preponderant role in the regulation of transcription, as chromatin organisation controls gene expression. Highly condensed chromatin does not allow DNA to be transcribed while less densely packed complexes are permissive for gene expression, stimulating it by recruiting transcription factors. The chromatin state depends on cellular epigenetic mechanisms such as histone modification and DNA methylation [11].

Because HIV-1 integrates its genome into the host genome, it was expected to be influenced by the chromatin environment. Indeed, research conducted in in vitro models showed that the presence of histones at certain sites of the HIV-1 genome is partly responsible for viral latency in $\mathrm{CD} 4^{+} \mathrm{T}$ cells [10]. Histone deacetylases (HDAC) remove acetyl groups from histones in order to enhance their interactions with DNA, which represses transcription. In several model systems, the inhibition of these enzymes activates viral gene expression through the recruitment of transcription factors to the viral genome [8].

HDAC inhibitors, such as valproic acid (VPA), vorinostat and panobinostat, were shown to increase production of HIV-1 RNA in in vitro models. However, VPA was quickly found to be ineffective in vivo [8]. The potential of vorinostat was also called into question after a study using patient cells found that this compound only minimally activated transcription [14]. It is now thought that the vorinostat-induced HIV1 RNA production observed in vitro might be caused by a different mechanism and will not be significant in vivo [7]. Panobinostat has recently been found to disrupt latency in vivo, but it did not reduce the number of latently infected cells [21].

Other proteins involved in epigenetic regulation, such as histone methyltransferases (HMT), DNA methyltransferases (DNMT), may also represent possible targets for the reactivation of latent virus [2]. Inhibition of DNMT has been studied in this context, but recent studies indicate that the role of DNA methylation in resting $\mathrm{T} \mathrm{CD} 4^{+}$cells may be less relevant than previously thought [14].

Upregulation of transcription factors: Signalling pathways involved in HIV-1 transcription can be targeted by many pharmacological compounds to trigger the production of latent virus [19].

Protein kinase $\mathrm{C}$ (PKC) agonists, such as prostratin and bryostatin-1, induce viral transcription by activating NF- $\kappa \mathrm{B}$, which, as previously mentioned, is a transcription factor that is absent from the nucleus in resting cells. Translocation of activated NF- $\kappa B$ to the nucleus mediated by the PKC pathway results in HIV-1 transcription [3]. The safety of these molecules is still a matter of debate, as PKC activation induces expression of many genes, possibly causing toxicity due to the release of cytokines [7]. Targeting specific PKC isoenzymes capable of inducing viral gene expression might be advantageous.

Disulfiram, a drug used in the treatment of chronic alcoholism, has been shown to induce HIV-1 gene expression through activation of a different signalling pathway that also leads to NF- $\mathrm{kB}$ activation [14].

Basal HIV-1 transcription is ongoing in infected cells if certain transcription factors, such as NF- $\mathrm{kB}$, are present in the cell nucleus [22], but still RNA polymerase II (RNAPII) is not able to originate complete mRNAs in the absence of other essential factors, as it is stalled by transcription repressors [19]. The HIV-1 protein Tat activates viral transcription by recruiting positive transcription elongation factor $\mathrm{b}(\mathrm{P}-\mathrm{TEFb})$ to an RNA hairpin structure called transactivation response element (TAR) present on the short viral transcripts produced by RNAPII [22]. P-TEFb phosphorylates this enzyme as well as the inhibitory transcription repressors, stimulating transcriptional elongation [20]. During latency, low levels of Tat and P-TEFb in the nucleus inhibit viral transcription.

A bromodomain protein, BRD4, competes with Tat for $\mathrm{P}-\mathrm{TEFb}$ binding at the HIV-1 promoter. BRD4 inhibitors such as JQ1 may reactivate latent virus, since they enhance Tat recruitment of $\mathrm{P}-\mathrm{TEFb}$ by dissociating BRD4 from the viral promoter [8]. Hexamethylene bisacetamide (HMBA) also activates a pathway that leads to the release of $\mathrm{P}-\mathrm{TEFb}$ from an inhibitory cytoplasmic complex, stimulating viral transcription via a Tat-independent mechanism [11]. The aforementioned host transcription repressors could also be interesting targets [11].

It remains unclear whether or not LRAs can produce a clinically significant reduction in the size of the latent reservoir. In fact, so far, they have not been shown to reduce it at all [23]. The main problem with research in this area is that in vitro models of latency may not reflect the behaviour of the latent reservoir in vivo, and most of these strategies have only been validated in the former [10]. The variability of responses of the several models to the same drug indicates that the process of latency is multifactorial and hard to mimic in vitro, since cell-specific factors are likely to have a role in latency that is very hard 
to replicate [4]. New studies seem to point to the inefficacy of many of the aforementioned compounds in activating HIV-1 transcription. Vorinostat, panobinostat, disulfiram and JQ1 all failed to induce viral outgrowth using patient-derived-infected cells, suggesting that none of these non-T-cell-activating compounds actually reactivate latent HIV1 , at least not individually [20]. Combinatorial treatment targeting multiple mechanisms and in several rounds might be necessary to induce reversal of latency [4].

In terms of safety, it is important to note that the modification of gene expression in host cells is likely to affect many other genes. Targeted approaches may be necessary to avoid off-target effects [24]. It should also be kept in mind that the reactivation of HIV-1 in sanctuary sites where cART cannot effectively prevent infection constitutes a risk for the patient. Improving antiretroviral therapy is fundamental if the "shock-and-kill" approach is to be used in the future. Targeting HIV-1 in sanctuary sites may be possible with the use of nanotechnology [2].

\section{Gene therapy}

The case of the Berlin patient was unprecedented and represented a landmark in HIV-1 cure research. Transplantation with donor cells resistant to infection by the most common strains of HIV-1 resulted in prolonged suppression of viraemia, which may or may not have been associated with reservoir depletion because of other factors. Although replicating this case is presumably impossible, it has laid the groundwork for the development of gene therapy strategies aimed at the discovery of a cure for HIV-1. The goal of gene editing techniques is to manipulate specific sequences in either the viral or the host cell genomes to induce changes in the pattern of protein expression that will interfere with a number of mechanisms involved in the viral life cycle [2], rendering the virus unable to successfully complete it.

Protein-based strategies: The use of zinc-finger nucleases (ZFNs) or other artificial sequence-specific nucleases is being explored as a strategy to disrupt CCR5 expression in $\mathrm{CD} 4^{+} \mathrm{T}$ cells, thereby making them resistant to infection by M-tropic HIV-1. ZFNs are restriction enzymes containing a zinc finger DNA-binding domain and a DNAcleaving domain. These enzymes can be used to target specific genes and create double-strand breaks in the cell DNA, which can subsequently be repaired by non-homologous end joining, a cell repair mechanism that is error-prone and introduces mutations that leave the gene nonfunctional. ZFNs that target the CCR5 gene block production of this co-receptor by the cell [9].

Both $\mathrm{CD}^{+} \mathrm{T}$ cells and HSCs can be treated with ZFNs [3]. By harvesting patient cells for gene therapy with ZFNs and subsequently reinfusing them into the patient's body, a population of resistant $\mathrm{CD} 4^{+}$ $\mathrm{T}$ cells is expected to proliferate, limiting viral replication and stunting viraemia in the absence of cART. This approach has the advantage of bypassing the need for a suitable donor and allogeneic transplantation and it has proven successful in mice, where it has resulted in a reduction of plasma viral RNA. Clinical trials where ZFNs are used to target $\mathrm{CD}^{+} \mathrm{T}$ cells are ongoing and primary results seem to indicate that engraftment is successful and treatment is well tolerated, improving $\mathrm{CD}^{+} \mathrm{T}$ cell count and conferring selective advantage to the resistant cells. A decline in blood HIV-1 DNA was also observed, suggesting a decrease in the reservoir. Although cART interruption led to viral rebound, clinical safety was demonstrated, fuelling further attempts to optimise this procedure [25].

Disruption of CXCR4 has also been tested and shown to provide resistance to T-tropic HIV-1. However, while CCR5 disruption does not result in serious adverse effects on immune function, the same has not yet been demonstrated for CXCR4 [9]. In addition, because nonmodified host cells are not eliminated by this technique, the latent reservoir will not be eradicated, and so a sterilising cure will not be achieved [10].

RNA-based strategies: Besides ZFNs, RNA-based strategies such as the use of ribozymes, small-interfering RNA (siRNA) and antisense RNA are other potential approaches to HIV-1 eradication. Ribozymes are catalytic RNAs designed to cleave specific sequences; siRNAs target specific mRNA sequences resulting in translational silencing or destruction of the mRNA [3].

An early attempt at using these tools for HIV-1 therapy consisted in delivering to HSCs harvested from patients a ribozyme targeting CCR5, a RNA decoy for Tat and a siRNA targeting expression of viral proteins [11]. However, the outcome of this study was not properly measured. The modified cells also presented with low levels of engraftment [15], resulting in a negligible effect on HIV-1 infection.

At the post-transcriptional level, latency can be maintained by inhibition of nuclear RNA export to the cytoplasm and inhibition of translation by microRNAs (miRNAs) [11]. A miRNA is a small noncoding single-stranded RNA molecule involved in RNA silencing and post-transcriptional regulation of gene expression. It has been shown that miRNAs in resting $\mathrm{CD} 4^{+} \mathrm{T}$ cells regulate viral expression and interact with viral mRNA, either causing its degradation or suppressing translation. Tat may also be implicated in the modulation of miRNA expression [11]. Agglomerates of miRNAs have been detected in resting cells, and not in active $\mathrm{CD}^{+}$cells. The use of anti-miRNA antisense RNA has been proposed as a strategy to reactivate resting cells, but this approach may cause toxicity in uninfected cells [2].

Provirus excision: Gene therapy technology may also be used to directly remove the provirus from infected cells. Very recent studies in HIV-1 gene editing strategies using the CRISPR/Cas9 system have successfully excised latent proviruses from infected cells in transgenic mice and rats, which resulted in a remarkable decrease in viral RNA in circulating blood and other tissues, demonstrating an effective suppression of viral expression [26]. This promising study suggests that in vivo elimination of HIV-1 DNA is possible and that the key for a sterilising cure may lie in the refinement of gene editing techniques.

\section{Permanent suppression by transcription inhibitors}

Considering the innumerable challenges surrounding reservoirpurging strategies, a perhaps more feasible approach has been proposed that consists in permanently driving the virus into a state of durable deep-latency, fully blocking residual viral replication from integrated proviruses and thereby preventing resurgence of viraemia and replenishment of the latent reservoir, even in the absence of antiretroviral therapy [22]. Benefits associated with this approach include the reduction of chronic immune activation and the possibility of controlling viraemia without cART, bypassing its long-term adverse effects.

Gene therapy approaches, particularly RNA-based strategies, have also focused on gene suppression, but they are not easy to make widely available. Research should not neglect the screening of compounds that can inhibit molecules involved in viral reactivation, such as Tat, TAR and P-TEFb. Blocking Tat and/or TAR would be ideal, as it would not affect cellular transcription. A drastic reduction in viral transcription could be achieved by disrupting Tat/TAR interaction. Inhibition of P-TEFb would have to focus on specifically inhibiting certain complexes, to not cause toxicity. Identification of small molecules that can be used with 
the purpose of fully suppressing HIV-1 in its reservoirs is still in its early stages [22].

\section{Immunotherapy}

Immune response to HIV-1 is defective and more harmful than helpful in fighting infection. Proper stimulation of the immune system to improve virus-specific responses could aid the host immune system in controlling viral replication in the absence of cART, mimicking the behaviour of ECs [4]. HIV-1-induced chronic inflammation could also be reduced with immune-based therapies. Coupled with latencyreversing strategies or by itself, immunotherapy can be crucial in the modulation of the immune response to HIV-1. Eradication strategies will probably require interventions at this level to assist in the elimination of the virus.

Therapeutic vaccines: One of the most widely discussed approaches to stimulate the immune system is therapeutic vaccination. Controlled exposure to viral antigens should induce CTL responses to conserved HIV-1 epitopes in patients who lack the protective HLA alleles that naturally confer this type of response in ECs [15]. Therapeutic vaccination has recently been shown to limit viral rebound after cART interruption [19], but not to a level where treatment discontinuation is possible [4]. Cytokines may be important adjuvants in therapeutic vaccines, but they have failed to improve cell function individually [8].

Downregulation of immunoregulatory molecules: The chronic inflammation observed in patients with HIV-1 does not equate with a good immune response to the virus. In fact, the persistent inflammatory environment induces immunoregulatory responses, which result in the upregulation of inhibitory receptors on the $\mathrm{T}$ cell surface that suppress their activation [27]. During HIV-1 infection, T cells gradually lose their effector function, leading to a state of immune exhaustion where they cannot effectively kill infected cells. The downregulation of immunoregulatory molecules, such as PD-1 and other markers of T cell exhaustion, could result in the reversal of this state. The inhibitory receptor PD-1 can be blocked with the use of antibodies [3].

Broadly neutralising monoclonal antibodies (bNAbs): Broadly neutralising monoclonal antibodies (bNAbs) can be used to target epitopes on the HIV-1 envelope protein. It remains unclear whether bNAbs can clear infected cells, but it is hoped that by eliminating free virus, antigen stimulation will decrease, resulting in the prevention of immune exhaustion and consequent improvement of CTL responses. $\mathrm{CD}^{+} \mathrm{T}$ cells should then be more efficient at eliminating infected cells. However, bNAbs may not be able to reach viral anatomical sanctuaries [27].

Other immune-based approaches: In a humanised mouse model, HIV-targeted immunotoxins in combination with cART demonstrated ability to kill cells with productive infection [14], reducing tissue levels of HIV-1 RNA.

Gene therapy may also be used in the context of immunotherapy to modify effector cells with receptors that enhance the detection of viral antigens [4].

\section{Concluding Remarks}

Antiretroviral therapy has been paramount in reducing HIVrelated mortality worldwide, but unfortunately it does not provide a cure for the infection, and so HIV/AIDS continues to be a major health issue, particularly in resource-limited countries.

The greatest hurdle in HIV-1 cure research is the viral latent reservoir, which has not yet been fully characterised. Further investigation focusing on the mechanisms behind HIV-1 latency remains a priority, as they may hold the key to identifying new targets and devising new strategies. The origin of a persistent viral load in patients under cART is still debatable. If cell types other than CD4+ T cells produce this residual viraemia, future strategies will have to focus on them as well. There is a clear need to design new models of latency that can better replicate the complex dynamics and environment of viral latency, as the development of effective approaches to eliminate the virus or force it into remission depends on having complete knowledge of viral behaviour in vivo.

Purging the latent reservoir to obtain a sterilising cure may have deleterious side effects and will require careful approaches coupled with therapies that enhance immune response to the virus. Alternatively, a growing hope is that a functional cure may be achieved with very early antiretroviral therapy or by employing strategies that aim to reinforce latency or control viral replication in the absence of cART, preventing further infection of susceptible cells. Gene therapy can be used for this purpose as well as for removal of the integrated provirus, and it has shown promising results in recent studies. Immune-based strategies are likely to be necessary to help the immune system recognise and remove infected cells, to effectively tackle the reservoirs.

Recent efforts made towards the discovery of an HIV-1 cure have generated optimism that therapies more effective than cART will one day be available. The development of more advanced technological methods will certainly continue to contribute to the progress in the area.

\section{References}

1. UNAIDS (2016) Global AIDS Update.

2. Le Douce V, Janossy A, Hallay H, Ali S, Riclet R, et al. (2012) Achieving a cure for HIV infection: Do we have reasons to be optimistic? J Antimicrob Chemother 67: 1063-1074.

3. Barton KM, Burch BD, Soriano-Sarabia N, Margolis DM (2013) Prospects for treatment of latent HIV. Clin Pharmacol Ther 93: 46-56.

4. Archin NM, Sung JM, Garrido C, Soriano-Sarabia N, Margolis DM (2014) Eradicating HIV-1 infection: Seeking to clear a persistent pathogen. Nat Rev Microbiol 12: 750-764

5. Wagner EK, Hewlett MJ, Bloom DC, Camerini D (2008) Basic Virology (3rd ed) Blackwell Publishing, Malden, MA 399-400.

6. Collier L, Oxford J (2006) Human Virology (3rd ed) Oxford University Press Oxford 179-183.

7. Siliciano JD, Siliciano RF (2014) Recent developments in the search for a cure for HIV-1 infection: Targeting the latent reservoir for HIV-1. J Allergy Clin Immunol 134: 12-19.

8. Passaes CP, Sáez-Cirión A (2014) HIV cure research: Advances and prospects. Virology 454-455: 340-52.

9. Chan CN, Dietrich I, Hosie MJ, Willett BJ (2013) Recent developments in human immunodeficiency virus-1 latency research. J Gen Virol 94: 917-932.

10. Blankson JN, Siliciano JD, Siliciano RF (2014) Finding a cure for human immunodeficiency virus-1 infection. Infect Dis Clin North Am 28: 633-650.

11. Van Lint C, Bouchat S, Marcello A (2013) HIV-1 transcription and latency: An update. Retrovirology 10: 67.

12. Lafeuillade A, Stevenson M (2011) The search for a cure for persistent HIV reservoirs. AIDS Rev 13: 63-66.

13. Lorenzo-Redondo R, Fryer HR, Bedford T, Kim EY, Archer J, et al. (2016) Persistent HIV-1 replication maintains the tissue reservoir during therapy. Nature 530: 51-56.

14. Sebastian NT, Collins KL (2014) Targeting HIV latency: Resting memory T cells hematopoietic progenitor cells and future directions. Expert Rev Anti Infect Ther 12: $1187-1201$. 
Citation: Pereira I, Luxo C, Matos AM (2016) Treatment of Human Immunodeficiency Virus-1: Current Challenges and Future Perspectives. J AIDS Clin Res 7: 603. doi:10.4172/2155-6113.1000603

Page 9 of 9

15. Durand CM, Blankson JN, Siliciano RF (2012) Developing strategies for HIV-1 eradication. Trends Immunol 33: 554-562.

16. Persaud D, Gay H, Ziemniak C, Chen YH, Piatak M Jr, et al. (2013) Absence of detectable HIV-1 viremia after treatment cessation in an infant. N Engl J Med 369: 1828-1835.

17. Rainwater-Lovett K, Luzuriaga K, Persaud D (2015) Very early combination antiretroviral therapy in infants: Prospects for cure. Curr Opin HIV AIDS 10: 4-11.

18. Frange P, Faye A, Avettand Fenoël V, Bellaton E, Descamps D, et al. (2016) HIV 1 virological remission lasting more than 12 years after interruption of early antiretroviral therapy in a perinatally infected teenager enrolled in the French ANRS EPF CO10 paediatric cohort: A case report. Lancet HIV 3: e49-e54.

19. Archin NM, Margolis DM (2014) Emerging strategies to deplete the HIV reservoir. Curr Opin Infect Dis 27: 29-35.

20. Dahabieh MS, Battivelli E, Verdin E (2015) Understanding HIV latency: The road to an HIV cure. Annu Rev Med 66: 407-421.

21. Rasmussen TA, Tolstrup M, Brinkmann CR, Olesen R, Erikstrup C et al. (2014)
Panobinostat, a histone deacetylase inhibitor, for latent-virus reactivation in HIV infected patients on suppressive antiretroviral therapy: A phase $1 / 2$, single group, clinical trial. Lancet HIV 1: e13-e21.

22. Mousseau G, Mediouni S, Valente ST (2015) Targeting HIV transcription: The quest for a functional cure. Curr Top Microbiol Immunol 389: 121-145.

23. Bullen CK, Laird GM, Durand CM, Siliciano JD, Siliciano RF (2014) New ex vivo approaches distinguish effective and ineffective single agents for reversing HIV 1 latency in vivo. Nat Med 20: 425-429.

24. Battistini A, Sgarbanti M (2014) HIV-1 latency: An update of molecular mechanisms and therapeutic strategies. Viruses 6: 1715-1758.

25. Wang CX, Cannon PM (2016) The clinical applications of genome editing in HIV. Blood 127: 2546-2552.

26. Kaminski R, Bella R, Yin C, Otte J, Ferrante P, et al. (2016) Excision of HIV-1 DNA by gene editing: A proof-of-concept in vivo study. Gene Ther.

27. Barouch DH, Deeks SG (2014) Immunologic strategies for HIV-1 remission and eradication. Science 345: 169-174. 